\title{
Intervención en el lenguaje con niños bilingües y sus familias: Una revisión
}

\section{Language Intervention with Bilingual Children and Their Families: A Review}

\section{RESUMEN}

Silvia Nieva

Doctora en Psicología

Universidad Castilla de La-Mancha

Lidia Rodríguez

Doctora en Pedagogía Universidad Castilla de La-Mancha

\section{Solenn Roussel}

Logopeda

Universidad Castilla de La-Mancha
Autor correspondiente:

Silvia Nieva

Toledo - España

Correo-e: silvia.nieva@uclm.es

Recibido: $12 / 04 / 2017$

Aceptado: 28/06/2017
La atención clínica y educativa que se ofrece a niños bilingües es actualmente un reto para los profesionales. Es necesario investigar sobre la conceptualización del bilingüismo, su naturaleza y su relación con los diferentes entornos de aprendizaje. El objetivo del presente trabajo es revisar la literatura científica con el fin de explorar el papel del entorno familiar y las competencias del fonoaudiólogo/logopeda para garantizar una intervención fonoaudiológica/logopédica funcional en población bilingüe. Para ello, se ha estudiado la información más representativa disponible sobre el enfoque naturalista de intervención en multilingüismo. Así, se pretende reflexionar sobre los modelos de intervención que mejor se adecúan a las necesidades específicas de los niños bilingües y el papel que puede adoptar la familia para contribuir al desarrollo comunicativo de los niños en contacto con varias lenguas.

En primer lugar, se ofrece una aclaración terminológica, así como una visión del marco naturalista de intervención. A continuación, se presentan la metodología y los resultados de la revisión efectuada para niños bilingües con diversidad funcional y alteraciones del lenguaje oral. Se incluyen también recomendaciones en relación a las competencias del fonoaudiólogo que trabaje con población bilingüe. Finalmente, se concluye con una propuesta de futuras líneas de investigación aplicada en este campo considerando los modelos naturalistas centrados en la familia para proponer su adaptación a las intervenciones realizadas con niños bilingües.

Palabras clave: bilingüismo, fonoaudiología, intervención fonoaudiológica, intervención naturalista, enfoque centrado en la familia, logopedia.

\section{ABSTRACT}

The clinical and educative response required for bilingual children continues to be a challenge for professionals. It is necessary to research the conceptualization of bilingualism, its nature, and its relationship with different learning environments. The purpose of this paper is to review the literature to explore the role of the family environment and the speech \& language therapist's (SLT) competence in guaranteeing a functional language intervention in the bilingual population. To this end, the most significant information available about multilingualism and the naturalistic intervention approach has been reviewed. With this evidence, the best models of bilingual intervention are presented, as well as a reflection on how families can contribute to improve the language development of children speaking different languages.

In the first section, a terminological clarification of bilingualism is given, alongside the naturalistic frame of intervention, followed by research developed with bilingual children with functional diversity and oral language disorders. In that sense, recommendations about competence of the Speech and Language Therapist working with bilingual special needs children are also included. Finally, this paper provides a suggestion of future applied research in this field on naturalistic family-centered approaches used in language intervention with bilingual children.

Keywords: bilingualism, family-centered approach, naturalistic intervention, Speech and Language Therapy, Speech and Language Therapy intervention. 


\section{Introducción}

Considerando los nuevos paradigmas socioculturales en una sociedad globalizada, el bilingüismo es un tema de creciente interés en la investigación (Peña, 2016). Para los profesionales de la fonoaudiología, la atención a los niños bilingües es actualmente un reto. Se precisa ampliar la evidencia sobre su naturaleza y su relación con los entornos de aprendizaje para entender sus implicaciones en la intervención y poder separar los trastornos de las diferencias atribuibles a factores externos y no necesariamente patológicos. La toma de decisiones de los profesionales debe estar orientada a una práctica basada en la evidencia científica para contribuir con efectividad en la mejora de la calidad de vida de los niños y sus familias.

En los entornos bilingües, la familia juega un papel especialmente importante en particular cuando la lengua del hogar es una lengua minoritaria y entran en juego factores culturales (Nieva, 2016). De acuerdo a lo anterior, el objetivo de esta revisión es enmarcar el fenómeno del bilingüismo en relación con la práctica fonoaudiológica dentro de los enfoques naturalistas, que incorporan a la familia de forma activa en el proceso de intervención.

En la conceptualización del bilingüismo existe un acuerdo en que un niño bilingüe es aquel que está en contacto con más de una lengua. Existen diferentes interpretaciones entre las que destacamos las que consideran el uso funcional (McLeod, Verdon \& Bowen, 2013) y las competencias no verbales y culturales (Harris, 2004). La diversidad terminológica y conceptual dificulta la publicación de una investigación homogénea y el conocimiento de la muestra a la que generalizar los resultados.

\section{Entornos bilingües desde un enfoque naturalista}

Son numerosos los niños que interactúan en entornos en los cuales intervienen distintas lenguas, bien sea por condiciones familiares, escolares o sociales (Pearson, 2007). Las combinaciones entre las lenguas usadas son tan diversas como las circunstancias individuales de cada niño y su familia. Como ejemplos, se puede mencionar la emigración a un nuevo país, la decisión de una escolarización bilingüe o la convivencia en una comunidad en la que coexisten varias lenguas. Es importante entender los antecedentes sociales, ya que la estimulación lingüística recibida por parte de la sociedad también influye en el grado de competencia en cada lengua (Hoff \& Core, 2013; Pearson, 2007; Thordardottir, Cloutier, Ménard, Pelland-Blais y Rvachew, 2015).

Así, en el bilingüismo, los entornos determinan el uso de cada lengua. El niño bilingüe se enfrenta a la tarea de desarrollar estrategias pragmáticas para poder adaptarse a cada contexto social. Por tanto, en esta revisión se destaca la importancia de ofrecer una intervención fonoaudiológica enfocada hacia el entrenamiento de las habilidades de uso, además de centrarse en los aspectos lingüísticos que requiere el empleo de una o dos lenguas. De este modo, los niños podrán llegar a ser tan competentes en sus lenguas de uso como lo exija cada entorno comunicativo.

Para comprender las necesidades comunicativas del niño bilingüe en su entorno natural, habrá que profundizar tanto en su desarrollo del lenguaje como en el rol de la familia y las mencionadas exigencias 
del entorno comunicativo.

El enfoque naturalista es una metodología emergente en el ámbito fonoaudiológico. Consiste en el conjunto de acciones terapéuticas usadas para apoyar el desarrollo del lenguaje y la comunicación en los entornos naturales dentro de las rutinas de interacción cotidianas (McWilliam, 2010). En él se interviene directamente con el niño, o de manera mediada con las personas clave implicadas en el desarrollo comunicativo, que suelen ser principalmente los padres, aunque también se aplica a otros entornos como el escolar (Dunst, Raab \& Trivette, 2011).

Este abordaje se basa en la construcción de la confianza y la competencia de los padres y su objetivo es fomentar el aprendizaje y "usar los intereses de los niños para involucrarlos y promover la adquisición de una comunicación funcional y significativa junto con la competencia lingüística" (Dunst, Raab \& Hamby, 2016, p.154). De esta manera, los cuidadores principales son partícipes del tratamiento. En colaboración con el profesional, toman decisiones para facilitar el desarrollo durante el curso de la evaluación y la intervención.

Esta metodología se basa en el enfoque ecológico del desarrollo humano (Bronfenbrenner, 1986), en modelos ecológicos en logopedia/fonoaudiología (De las Heras y Rodríguez, 2015) y otros enfoques clásicos socio-interaccionistas (Bruner, 1986) que consideran al niño como parte del sistema familiar, que es interdependiente entre sí y donde existe una influencia bidireccional con los entornos en los que se desenvuelve.

El enfoque que incluye a la familia en el tratamiento se distingue de otros planteamientos usados tradicionalmente en fonoaudiología centrados en el terapeuta. Watts-Pappas, McLeod y McAllister (2009) introducen variaciones en el grado de implicación de la familia, desde: 1) un modelo de "familia como colaboradora", en el que el terapeuta toma las decisiones aunque puede tener en cuenta sugerencias de la familia, 2) un modelo de "familia como apoyo", en el que esta participa en algunas etapas de la intervención para generalizar aprendizajes y aplicar pautas, hasta llegar a 3) un modelo "centrado en la familia" en el que la familia toma decisiones sobre la intervención y participa de forma activa en el diseño de la misma y en su progreso.

\section{Método}

A continuación, se presentan las distintas estrategias de búsqueda empleadas para poder responder a los objetivos de la revisión, así como los principales resultados obtenidos en la revisión bibliográfica.

\section{Criterios de inclusión y exclusión}

Se incluyeron artículos de revistas con revisión por pares, publicados entre 2004 y la actualidad, sobre el desarrollo lingüístico, desarrollo cognitivo, competencias del fonoaudiólogo, evaluación e intervención fonoaudiológica, intervención naturalista en fonoaudiología e intervención naturalista en bilingüismo (véanse los términos utilizados en inglés en Tabla 1). En cuanto a la población estudiada, ya que el propósito del trabajo se centra en la atención fonoaudiológica de la 
población infantil bilingüe, los artículos elegidos aportan conclusiones sobre el abordaje terapéutico en niños con trastornos del lenguaje en edades

Tabla 1

Términos según criterios de inclusión

\begin{tabular}{lll}
\hline Población y edad & Tipo de intervención & Entorno de intervención \\
\hline bilingu* & assessment & caregiver \\
dual language learner & clinical intervention & family-centred \\
second language learner & cognitive development & family therapy \\
language disorder & language development & home-based \\
children & language treatment & natural environment intervention \\
toddler & speech-language therapy & parent-based \\
preschooler & speech-language pathologist & parent coaching \\
\hline
\end{tabular}

\section{Métodos de búsqueda}

Se procedió a una búsqueda en inglés, español y francés, aplicada en diferentes bases de datos: Medline, Psicodoc, PsychINFO, PubMed, PubPsych y Google Académico. Por otro lado, se realizó una búsqueda manual a través de las páginas de asociaciones internacionales de fonoaudiología.

\section{Resultados}

A continuación, se presentan los resultados más relevantes de la revisión sobre bilingüismo en poblaciones con diversidad funcional y, por último, las competencias del fonoaudiólogo en intervención con niños bilingües.

Se manejaron, en total, 89 referencias de revisiones, estudios empíricos y artículos teóricos.

Los artículos seleccionados tienen distintas características. La mayor parte de las publicaciones se centran principalmente en las lenguas infantiles y escolares hasta los seis años. Los modelos estudiados fueron clínicos y naturalistas. 
posibles quedaba muy lejos de las motivaciones de los investigadores.

En este momento existen cada vez más estudios centrados en la adquisición bilingüe en niños con diversidad funcional y alteraciones del lenguaje, aunque esta investigación está localizada en áreas geográficas muy limitadas, y aún no recoge las idiosincrasias de las distintas poblaciones bilingües en el mundo.

La dificultad de este tipo de investigaciones centradas en la diversidad funcional estriba, por una parte, en la homogeneización de las muestras según el grado de discapacidad y el nivel de desarrollo y, por otra parte, en las variaciones individuales, y en el tamaño del impacto de los factores ambientales (del mismo modo que cualquier investigación sobre adquisición y desarrollo del lenguaje infantil).

En el caso de los bilingües, se añade la variación de los entornos de uso de las lenguas, tanto en cantidad como en calidad de exposición.

Entendemos la investigación como el estudio de las variables que se relacionan con diferentes fenómenos en la búsqueda de modelos explicativos, que se presentan como posibilidades, no necesariamente como verdades, pudiendo obtenerse evidencia que sirva para fundamentar nuevas posibilidades. Por tanto, los resultados de las investigaciones que aquí se exponen son las posibilidades que actualmente presentan evidencia empírica. Los profesionales que basan su práctica clínica en resultados de investigación tienen la responsabilidad de actualizar y contrastar la información que reciben.

En relación con la fonoaudiología como disciplina, se encuentra que la información disponible sobre bilingüismo parte de una interpretación individual, con muestras pequeñas e inconsistentes (debido a la dificultad de homogeneizar una muestra por la idiosincrasia de las personas bilingües, su historia vital y sus entornos de experiencia).

Es fundamental ampliar la evidencia empírica para extenderla hacia un enfoque grupal de referencia y para poder comprobar la eficacia de programas de intervención de una forma consistente. En tanto este objetivo no sea posible, la toma de decisiones en la evaluación y tratamiento fonoaudiológico debe estar predominantemente centrada en la persona y en su entorno.

Así, se han encontrado estudios que avalan el uso de una metodología de intervención naturalista, aunque aún son escasos (Del Río, 2006; GalvanBovaira y Del Río, 2010; Escorcia, García, Orcajada y Sánchez, 2016; Girolametto \& Weitzman, 2009; Gràcia, Ausejo y Porras, 2010; Lozano, Galián-Conesa \& Cabello-Luque, 2009; Rakap y Rakap, 2014).

Actualmente, los resultados de la investigación previa, tal y como declaran Marinova-Todd y Mirenda (2012), no muestran un impacto negativo del bilingüismo en el desarrollo de las alteraciones del lenguaje asociadas con Trastorno Específico del Lenguaje, Síndrome de Down o los Trastornos del Espectro Autista. Los niños que, dentro de estos grupos, presentan diversidad funcional tienen la capacidad de llegar a ser bilingües.

\section{Trastornos del Espectro Autista (TEA)}

En los niños con Trastornos del Espectro Autista (TEA), se ha observado una emergencia tardía del lenguaje en comparación con los niños de desarrollo típico. De hecho, entre el 10 y el $20 \%$ de los niños 
TEA puede no llegar a adquirir habla o lenguaje funcional (Mirenda et al., 2013).

Distintas investigaciones han comparado niños TEA bilingües con niños TEA monolingües. Hambly y Fombonne (2012) no encuentran diferencias entre ambos grupos en habilidades lingüísticas en 9 lenguas distintas, ni en bilingües secuenciales ni simultáneos, excepto en familias con madres no nativas, aportando evidencia que refuerza la necesidad de un input de calidad. El estudio de Ohashi et al., (2012) a partir de medidas receptivas, de producción y funcionales, también concluye que ambos grupos son equivalentes.

A su vez, se encuentran trabajos que consideran aspectos multimodales. Así, Valicenti-McDermott et al., (2013), examinando habilidades receptivas, expresivas y gestos comunicativos, vuelven a concluir que ambos grupos cuentan con el mismo nivel de desarrollo verbal y no verbal, aunque declaran que el grupo bilingüe utiliza más gestos.

Yang (2011) encuentra que, en población bilingüe de inglés/mandarín, los bilingües con TEA tienen más dificultades que los bilingües normotípicos en habilidades narrativas, mientras que se siguen obteniendo medidas equivalentes en bilingües con TEA y monolingües con TEA en otros aspectos gramaticales.

En resumen, a pesar de que los estudios no son muy numerosos, todos muestran resultados concurrentes, no encontrándose estudios que indiquen que el bilingüismo tiene efectos negativos en esta población (De Oliveira, 2015; Drysdale, Van Der Meer \& Kagohara, 2015). No obstante, no puede olvidarse que estos estudios emplean en su mayoría metodología cualitativa, basada en observación, narración y entrevistas.

\section{Trastorno Específico de Lenguaje (TEL)}

Tal y como constatan diferentes autores, entre ellos Ebert, Kohnert, Pham, Disher y Payesteh (2014), los niños bilingües con TEL presentan dificultades que se hacen patentes en todas sus lenguas.

Los trabajos que comparan las habilidades cognitivas y lingüísticas de niños bilingües con TEL y niños monolingües con TEL, encuentran resultados equivalentes en ambos grupos en lo que refiere al tipo de errores (Jacobson \& Schwartz, 2005), a las dificultades en algunas tareas de procesamiento no específicamente lingüístico (Ebert et al., 2014) y en competencia morfosintáctica (Paradis, 2007). No obstante, los resultados no son tan consistentes como en el TEA, ya que se encuentran estudios que muestran resultados inferiores para bilingües con TEL (Orgassa \& Weerman, 2008).

En cuanto a los efectos de la intervención, en uno de los escasos estudios sobre TEL que investiga acerca de la eficacia de distintos programas de intervención (Ebert et al., 2014) se evalúan tratamientos en diferentes lenguas y un tratamiento únicamente en estrategias cognitivas. Como resultado, se encuentran mejoras en todos los tratamientos, pero escasa mejoría en la lengua minoritaria.

Otro estudio que compara a niños bilingües TEL con niños bilingües normotípicos encuentra que en las variables observadas: procesamiento fonológico, atencional y del lenguaje, los niños bilingües TEL presentan limitaciones de procesamiento que se relacionan con dificultades de escritura (AguilarMediavilla, Buil-Legaz, Pérez-Castelló, Rigo-Carratalà 
\& Adrover-Roig, 2014).

Esta población es actualmente objeto de numerosas investigaciones emergentes, por lo que se espera con el tiempo se cuente con evidencia más concluyente.

\section{Síndrome de Down (SD)}

Los niños con Síndrome de Down muestran un desarrollo del lenguaje similar a los niños de desarrollo típico. No obstante, algunos estudios han informado de un desfase mayor en comprensión y expresión y de que su memoria de trabajo es menor (Chapman \& Hesketh, 2000).

Según los expertos, el bilingüismo no afecta negativamente a los niños con Síndrome de Down ni retrasa su desarrollo lingüístico (Edgin, Kumar, Spano \& Nadel, 2011; Feltmate \& Kay-Raining, 2008; Vallar y Papagno, 1993).

Se ha mostrado una relación positiva entre la edad mental y sus habilidades en la lengua dominante para la comprensión de vocabulario. A su vez, esta relación positiva se encuentra en la lengua no dominante en medidas de desarrollo sintáctico como la Longitud Media de Emisión (Kay-Raining, Cleave, Trudeau, Thordardottir, Sutton \& Thorpe, 2005).

Al comparar a bilingües con Síndrome de Down con monolingües con Síndrome de Down no se han encontrado diferencias en vocabulario receptivo ni en morfosintaxis (Kay-Raining et al., 2005; Feltmate \& Kay-Raining, 2008).

\section{Discusión}

La orientación para la práctica fonoaudiológica en entornos bilingües es un campo de investigación reciente. Durante varios años se han discutido las posibles consecuencias negativas del bilingüismo para el desarrollo de los niños.

Las investigaciones sobre el bilingüismo en personas con diversidad funcional sugieren que es preferible realizar comparaciones entre bilingües (con alteraciones del lenguaje y normotípicos) y entre niños bilingües que presentan alteraciones y monolingües con el mismo diagnóstico, por las particularidades de los bilingües y porque los resultados de las investigaciones recientes van en la dirección de que las dificultades de los niños bilingües con alteraciones son las de su condición, sea éste bilingüe o monolingüe, no existiendo una dificultad inherente al bilingüismo. Argumentan que en muchas ocasiones se confunden las diferencias individuales con las dificultades.

Existe cierta evidencia a favor de la falta de consecuencias negativas para el desarrollo fruto de la condición de ser bilingüe (tanto en población típica como en población con diversidad funcional) y se cuenta con recomendaciones de introducir y mantener la estimulación bilingüe como beneficio cognitivo, social, y lingüístico en atención temprana. Sin embargo, no están claras las prácticas de instrucción, ni las técnicas terapéuticas adecuadas para los niños bilingües que presentan dificultades tal y como reivindican los profesionales implicados (Buysse, Peisner-Feinberg, Páez, Hammer \& Knowles, 2014).

No obstante, varias asociaciones internacionales proponen orientaciones para los profesionales, como la American Speech-Language Association o la Internacional Association of Logopedics and 
Phoniatrics (ASHA, 2012; Fredman, 2006), no obstante, los criterios utilizados para esas recomendaciones terapéuticas no están aún claros y, en ocasiones, parecen alejados de la realidad terapéutica o no están adaptados para todos los entornos culturales de cada país.

El problema fundamental consiste en construir un marco teórico conceptual claro que sirva de guía para la práctica clínica y educativa. Con el fin de mejorar la intervención con niños bilingües se discute el uso de métodos clínicos frente a la adaptación de un enfoque naturalista, aunque este debate es aún emergente. Con el interés de proponer soluciones a esta problemática, en este artículo se proponen los modelos naturalistas como modelo teórico a valorar en la evaluación e intervención en edades tempranas con poblaciones bilingües.

Para poder ofrecer una atención de calidad a los niños bilingües y a sus familias, el fonoaudiólogo debe entender las necesidades específicas de los niños que se desarrollan en contacto con varias lenguas y adquirir ciertas competencias. En su práctica profesional, se enfrenta a diversas dificultades cuando no habla la lengua nativa del niño, no existen pruebas estandarizadas en las lenguas minoritarias y/o desconoce los procesos de adquisición y/o las convenciones culturales que afectan a la interacción en las lenguas implicadas.

Los fonoaudiólogos que trabajan en entornos bilingües asumen que desarrollan su actividad en un contexto cambiante, tanto en cuestiones relativas a la lengua, como en la cultura y en los aspectos emocionales.

Así, las necesidades emocionales de los niños multilingües han de ser también atendidas, ya que, en caso contrario, y como señalan McAllister y Lincoln (2004), el lenguaje puede verse obstaculizado. Desde el enfoque naturalista de intervención y el enfoque centrado en la persona y la familia, la gestión emocional debería ser considerada y entrenada de manera explícita.

\section{Conclusiones}

En esta revisión se recoge una muestra representativa y actualizada de estudios disponibles sobre el desarrollo de los niños bilingües con y sin alteraciones del lenguaje, a la vez que se ha tratado de exponer varios de los planteamientos actuales en relación con la atención fonoaudiológica en esta población. Las evidencias recogidas sugieren la idoneidad de un planteamiento bilingüe tanto en el proceso de evaluación como en el de intervención. Asimismo, destacan las dificultades que encuentran los profesionales para adaptarse a estas recomendaciones cuando no tienen acceso a las lenguas que emplea el niño, o no disponen los recursos suficientes para evaluar e intervenir.

Los niños bilingües utilizan diferentes lenguas en función de cada contexto de interacción, por lo que una metodología de intervención basada en el entorno natural podría ser indicada para cubrir las necesidades de cada familia. El profesional puede considerar la inclusión de la familia en el tratamiento para estimular el lenguaje de forma directa y en las rutinas del niño y valorar su papel como los comunicadores más frecuentes con el niño. Además, podría apoyar la comunicación familiar, reduciendo factores de estrés ante situaciones difíciles, al dotar 
al entorno de herramientas comunicativas

funcionales para cada interacción.

Para que la práctica fonoaudiológica pueda adaptarse a las necesidades de los niños bilingües, es importante que los fonoaudiólogos amplíen la investigación y se formen sobre los procesos de desarrollo específicos de la población bilingüe y sus implicaciones socioculturales. De esa manera, tendrán a su disposición herramientas para proponer planes de intervención eficaces, involucrando al entorno según criterios funcionales y adaptados a cada persona.

\section{Referencias}

Aguilar-Mediavilla, E., Buil-Legaz, L., Pérez-Castelló, J. A., Rigo-Carratalà, E., \& Adrover-Roig, D. (2014). Early preschool processing abilities predict subsequent reading outcomes in bilingual Spanish-Catalan children with Specific Language Impairment (SLI). Journal of communication disorders, 50, 19-35.

American Speech-Language Hearing Association (ASHA). (2012). Bilingual service delivery. ASHA Practice Portal. Recuperado el 18 de febrero de 2015, de http://www.asha.org/PRPSpecificTopic. aspxfolderid=8589 935225ysection=Key_Issues

Buysse, V., Peisner-Feinberg, E., Páez, M., Hammer, C. S., \& Knowles, M. (2014). Effects of early education programs and practices on the development and learning of dual language learners: A review of the literature. Early Childhood Research Quarterly, 29, 765-785.

Bronfenbrenner, U. (1986). Ecology of the family as a context for human development: Research perspectives. Developmental Psychology, 22(6), 723-742.

Bruner, J. (1986). El habla del niño: Aprendiendo a usar el lenguaje. Barcelona: Paidós.

Chapman, R. S., \& Hesketh, L. J. (2000). Behavioral phenotype of individuals with Down syndrome. Developmental Disabilities Research Reviews, 6(2), 84-95.
De las Heras, G., y Rodríguez, L. (2015). Guía de Intervención Logopédica en Dislalias. Madrid: Síntesis.

De Oliveira, Ê. (2015). Una revisión de la literatura sobre el bilingüismo entre los niños diagnosticados con Trastornos del Espectro Autista. Revista Chilena de Fonoaudiología, 14, 33-44.

Del Río, M. J. (2006). Consideraciones sobre el uso de los procedimientos naturalistas para la intervención en fonoaudiología. Revista de Logopedia, Foniatría y Audiología, 26(3), 139-145.

Drysdale, H., Van Der Meer, L., \& Kagohara, D. (2015). Children with Autism spectrum disorder from bilingual families: A systematic review. Review Journal of Autism and Developmental Disorders, 2(1), 26-38.

Dunst, C., Raab, M. \& Trivette, C. (2011). Characteristics of naturalistic language intervention strategies. Journal of Speech-Language Pathology and Applied Behavior Analysis, 5(1), 8-16.

Dunst, C. J., Raab, M., \& Hamby, D. W. (2016). Interestbased everyday child language learning. Revista de Logopedia, Foniatría y Audiología, 36(4), 153-161.

Ebert, K., Kohnert, K., Pham, G., Disher, J. \& Payesteh, D. (2014). Three treatments for bilingual children with primary language impairment: examining cross-linguistic and cross-domain effects. Journal of Speech, Language and Hearing Research, 57(1), 172-186.

Edgin, J. O., Kumar, A., Spano, G., \& Nadel, L. (2011). Neuropsychological effects of second language exposure in Down syndrome. Journal of Intellectual Disability Research, 55(3), 351-356.

Escorcia, C., García, F., Orcajada, N., y Sánchez, M. (2016). Perspectiva de las prácticas de atención temprana centradas en la familia desde la logopedia. Revista de Logopedia, Foniatría y Audiología, 36(4), 170-177.

Feltmate, K., \& Kay-Raining, E. (2008). Language Learning in Four Bilingual Children with Down Syndrome: A Detailed Analysis of Vocabulary and Morphosyntax L'apprentissage du langage chez quatre enfants bilingues atteints du syndrome de Down: une analyse. Revue Canadienne d'orthophonie et d'audiologie-Vol, 32(1), 7.

Fredman, M. (2006). Recommendations for working with 
bilingual children-prepared by the multilingual affairs committee of IALP. Folia Phoniatria et Logopaedica, 58(6), 456-464.

Galvan-Bovaira, M. J. y Del Río, M. J. (2010). Intervención naturalista. Revista de Logopedia, Foniatría y Audiología, 30(4), 165-166.

Girolametto, L. \& Weitzman, E. (2009). Working with families of young children with communication and language impairments: intervention. En N. Watts-Pappas y S. McLeod (Eds.), Working with families in speech-language pathology (pp. 131-170). San Diego: Plural Publishing.

Gràcia, M., Ausejo, R. \& Porras, M. (2010). Intervención temprana en comunicación y lenguaje: colaboración con las educadoras y familias de dos niños. Revista de Logopedia, Foniatría y Audiología, 30(4), 186-195.

Hambly, C., \& Fombonne, E. (2012). The impact of bilingual environments on language development in children with autism spectrum disorders. Journal of Autism and Developmental Disorders, 42(7), 1342-1352.

Harris, K. (2004). Speech-language pathologists' professional efficacy beliefs about assessing the language skills of bilingual/bicultural/bidialectal students. [Tesis Doctoral, University of South Florida].

Hoff, E. \& Core, C. (2013). Input and language development in bilingually developing children. Seminars in Speech and Language, 34(4), 215-226.

Jacobson, P. F., \& Schwartz, R. G. (2005). English past tense use in bilingual children with language impairment. American Journal of Speech-Language Pathology, 14(4), 313-323.

Kay-Raining, E., Cleave, P., Trudeau, N., Thordardottir, E., Sutton, A., \& Thorpe, A. (2005). The language abilities of bilingual children with Down syndrome. American Journal of Speech-Language Pathology, 14(3), 187-199.

Lozano, E., Galián-Conesa, M. D. \& Cabello-Luque, F. (2009). Family intervention in children with language disabilities: a review. Electronic Journal of Research in Educational Psychology, 7(3), 1419-1448.

Marinova-Todd, S. H., \& Mirenda, P. (2012). Language and communication abilities of bilingual children with Autisim Spectrum Disorders. En: J. Patterson y Barbara L. Rodriguez
(Eds.), Multilingual perspectives on child language disorders. Bristol, UK: Multilingual Matters.

McAllister, L., \& Lincoln, M. (2004). Clinical Education in Speech-Language Pathology. Nueva Zelanda: John Wiley y Sons.

McLeod, S., Verdon, S. \& Bowen, C. (2013). International aspirations for speech-language pathologists' practice with multilingual children with speech sound disorders: development of a position paper. Journal of Communication Disorders, 46(4), 375-387.

McWilliam, R. A. (2010). Assessing families' needs with the Routines-Based Interview. En R. A. McWilliam (Ed.), Working with families of young children with special needs (pp. 27-59). New York: The Guilford Press.

Mirenda, P., Smith, I. M., Volden, J., Szatmari, P., Bryson, S., Fombonne, E., \& Thompson, A. (2013). How many children with autism spectrum disorder are functionally nonverbal [Comunicación presentada en: International Meeting for Autism Research. San Sebastian.].

Nieva, S (2016). Competencia cultural en la práctica logopédica con familias de origen cultural y lingüísticamente diversos. En M. Martín-Aragoneses, y R. López Higues (Coords.). Claves de la Logopedia del Siglo XXI (pp. 55-71). Madrid: UNED.

Ohashi, J.K., Mirenda, P., Marinova-Todd, S., Hambly, C., Fombonne, E., Szatmari, P., ... \& Thompson, A. and the Pathways in ASD Study Team (2012). Comparing early language development in monolingual and bilingualexposed young children with autism spectrum disorders. Research in Autism Spectrum Disorders 6, 890-897.

Orgassa, A., \& Weerman, F. (2008). Dutch gender in specific language impairment and second language acquisition. Second Language Research, 24(3), 333-364.

Paradis, J., Schneider, P. \& Duncan, T. S. (2013). Discriminating children with language impairment among English-language learners from diverse first-language backgrounds. Journal of Speech, Language and Hearing Research, 56(3), 971-981.

Pearson, B. Z. (2007). Social factors in childhood bilingualism in the United States. Applied Psycholinguistics, 28(3), 399-410. 
Peña, E. (2016). Supporting the home language of bilingual children with developmental disabilities: From knowing to doing. Journal of Communication Disorders. 63, 85-92.

Rakap, S. \& Rakap, Se. (2014). Parent-implemented naturalistic language interventions for young children with disabilities: a systematic review of single-subject experimental research studies. Educational Research Review, 13, 35-51. Recuperado de: http://daneshyari.com/article/preview/355097.pdf

Romañach, J., \& Lobato, M. (2005). Diversidad funcional, nuevo término para la lucha por la dignidad en la diversidad del ser humano. Foro de vida independiente, 5, 1-8.

Thordardottir, E., Cloutier, G., Ménard, S., Pelland-Blais, E., \& Rvachew, S. (2015). Monolingual or bilingual intervention for primary language impairment? a randomized control trial. Journal of Speech, Language, and Hearing Research, 58(2), 287-300.

Valicenti-McDermott, M., Tarshis, N., Schouls, M., Galdston, M., Hottinger, K., Seijo, ... \& Shinnar, S. (2013). Language differences between monolingual English and bilingual English-Spanish young children with autism spectrum disorders. Journal of Child Neurology, 28(7), 945948.

Vallar, G., \& Papagno, C. (1993). Preserved vocabulary acquisition in Down's syndrome: The role of phonological short-term memory. Cortex, 29(3), 467-483.

Watts-Pappas, N., McLeod, S. \& McAllister, L. (2009). Models of practice used in speech-language pathologists' work with families. En N. Watts-Pappas y S. McLeod (Eds.), Working with families in speech-language pathology (pp. 1-38). San Diego: Plural Publishing.

Yang, S. K. T. (2011). Narrative abilities in bilingual children with autism. [Tesis Doctoral, University of British Columbia, Vancouver, Canada]. 